\title{
Optimal Algorithm of Full Focus Array Based on Sparse Matrix
}

\author{
Donghan Wang ${ }^{1,}$,, Lingxuan Chen ${ }^{2}$, Shijun Que ${ }^{1,}$,, Yunhe Gao ${ }^{1}$ \\ ${ }^{1}$ School of Electrical and Electronic Engineering,Tiangong University, Tianjin 300401, China \\ ${ }^{2}$ College of Engineering Science and Technology, Shanghai Ocean University, Shanghai 201306, China \\ *Corresponding Author.
}

\begin{abstract}
Ultrasonic phased array all-focus imaging algorithm has the advantages of high accuracy and full range dynamic focusing, but the existing imaging time-consuming problem limits its practical industrial application. In order to meet the requirements of real-time imaging and clear and distinguishable image defects in all-focus array ultrasound scanning. In this paper, a sparse optimization model of TFM phased array is established. Based on the Fermat principle, the delay time of each element under two layers of media is calculated, and a two-layer media phased array all-focus imaging algorithm is established, using minimal redundancy. The sparse design of the MRLA is carried out, and the all-focus imaging and sparse all-focus imaging experiments of the two-layer medium are carried out, and the influence of the sparse emission array on the defect quantitative accuracy and the calculation efficiency of the all-focus algorithm is discussed. The final optimized sparse array is compared with the sound field beam pattern of the full array, the smallest redundant array, and the genetic algorithm optimized array, and an all-focus scanning environment is established based on the array. Scanning images can not only obtain the maximum amount of information under minimum redundancy, but also have good sidelobe characteristics. At the same time, the array is sparse, which greatly improves the efficiency of all-focus scanning, and provides a certain reference value for the research of all-focus imaging technology.
\end{abstract}

Keywords: ultrasonic phased array; sparse matrix; all-focus algorithm.

\section{Theories and basic concepts}

Compared with single-probe ultrasonic detection, ultrasonic phased array detection has the advantages of fast detection speed, high sensitivity, and adaptability to complex objects. It has been widely used in pressure-bearing and other special equipment detection[1]. Compared with the conventional ultrasonic phased array detection, based on the all-focus ultrasonic phased array imaging, by post-processing the full matrix data of the transducer array, it can realize the convergence of any point in the measured area, which greatly improves the image resolution, Has a very good application prospect[2 3].

HOLMES et al. proposed the concept of full matrix capture (Full matrix capture, FMC) and a full matrix data model, and established a total focus method (TFM) imaging method based on full matrix data[5]. The full-matrix TFM imaging method has been successfully applied to the defect detection of important components and materials such as pressure pipeline welds, nuclear power plant welding components, carbon fiber composite materials, and so on. This type of research focuses on the detection of single-layer media, and the transducer is directly coupled with the workpiece. Therefore, it is of important application value to study this situation, that is, the all-focus imaging characteristics of the medium[6 78 8].

In addition, due to the large amount of full-matrix imaging data and time-consuming calculations, it limits the use of some industrial sites. To solve this problem, on the one hand, we can reduce the computing time by improving the hardware structure, such as using GPU and multi-field programmable gate array parallel computing. On the other hand, improving the calculation efficiency of the algorithm on the basis of ensuring the imaging quality has also become an important research content[9]. Both wavenumber domain imaging and migration techniques can significantly improve the post-processing speed of full matrix data. However, because the spatial sampling rate is affected by the interpolation function, it is easy to cause artifacts, and the algorithm principle is complex and

ISSN: 0010-8189

(C) CONVERTER 2021

www.converter-magazine.info 
difficult to apply to more than two layers of media. Sparse array elements can improve the imaging efficiency of phased arrays. PENG and others use sparse arrays to increase the effective aperture method to reduce computing time, but they are only used in single-layer media, and the sound field changes after sparse arrays are not considered, which affects the imaging quality[10] .

Based on the single-layer media full-focus algorithm, this paper calculates the delay time of each element in the media full-focus imaging based on the Fermat principle, and realizes the full-focus imaging during wedge detection. Based on the uniformity of the effective aperture of the array based on the equivalent point spread function, the weight function of the receiving element in the sparse array is studied, the sparse matrix-based media full-focus imaging algorithm is established, and the sparse matrix full-focus imaging and the full-matrix full-focus imaging are quantitatively analyzed Imaging accuracy and algorithm calculation efficiency of imaging[11 12].

\section{Research status at home and abroad}

\subsection{FMC/TFM research status}

Caroline Holmes of the University of Bristol, UK, first introduced the concept of ultrasonic phased array full matrix capture (Full matrix capture, FMC) in 2005, established a theoretical model of array FMC data acquisition, and discussed the total focus method based on FMC data (Total focus) [13]. TF city method, linear B-scan, focused B-scan, fan-shaped B-scan mathematical model and array performance indicator, Array performance Indicator, APD, and compared the detection performance of TFM method and conventional B-scan, and pointed out the calculation efficiency of FMC/TFM Very low[14]. In 2008, Alan et al. reported that the frequency domain wavenumber algorithm was used to improve the calculation efficiency of the FMCITFM detection method, and further improved the MC data acquisition model. They pointed out that the calculation efficiency of the wavenumber algorithm is theoretically $\mathrm{N}$ times that of the TFM algorithm, where $\mathrm{N}$ It is the number of elements in the array. At the same time, the wavenumber algorithm has stricter mathematical limitations than TFM[15]. The implementation process is very complicated, and the selected frequency domain interpolation function will greatly affect the quality of imaging. Moreau et al. pointed out in 2009, because The ultrasonic propagation speed in the specimen is limited, and there must be a minimum interval between two consecutive excitations, which limits the problem of acquisition speed. Based on this problem, the sparse matrix based on the far-field approximate effective aperture technology is discussed. LFM method and constant source Method (Common Source Method, CS1V) [16 17]. In 2010, Moreau et al. further discussed the wave number algorithm and the TFM imaging method using a defined sparse transmission matrix. The article pointed out that limiting the transmission period can reduce the acquisition and post-processing time, and demodulate the signal. The baseband can further reduce the postprocessing time. The use of a sparse transmission matrix can not only reduce the data acquisition and postprocessing time, but also the dynamic range of imaging accuracy[18]. The literature also points out that TFM cannot reach the frame rate of 1FPS, but the wave number algorithm is The frame rate of 2.SFPS can be reached under the fast post-processing method. Zhang et al. discussed and analyzed the influence of the inconsistency of array element parameters, such as amplitude, phase, and delay, on TFM imaging in 2011[19]. Paul and Zhang et al. respectively in 2008 And the introduction of the vector total focus method in 2010, ctor Total Focusing Method. VTF is used to evaluate the direction and reflection characteristics of scatterers and TFM imaging detection in nonuniform multilayer media. Caroline Holmes et al. proposed a Scanned Array-based post-processing method in 2008 to improve the effective aperture during static inspection and increase the angle range of the probe to improve imaging and defect location performance. SutclifFe et al. reported in 2012 that for the all-focus imaging method based on FMC data acquisition, the use of synthetic aperture technology can achieve higher resolution, but the imaging detection efficiency will be lower, and the real-time performance will be worse. The virtual source aperture is introduced., VSA) synthetic aperture method, and tested and compared the processing speed parameters of the FMC/TFM method, synthetic aperture focusing method, and virtual source aperture method. The results show that the virtual source method not only doubles the processing speed, but also maintains a good performance. The horizontal resolution and signal-to-noise ratio[19 20].

From the above analysis, it can be seen that the current key issues in the research and application of FMC-based

ISSN: 0010-8189

(C) CONVERTER 2021

www.converter-magazine.info 
imaging algorithms are in two aspects: First, the research on the key issues of actual detection applications based on FMC real-time high-resolution imaging; Research on imaging algorithms[21].

\section{2. Research status of sparse array design}

The all-focus algorithm needs to collect complete full-matrix data for offline post-processing[22]. Due to the large amount of full-matrix data, it takes a long time for all-focus imaging, which cannot achieve real-time highresolution detection in industrial applications. Aalan J. uses synthesis in radar Aperture focusing imaging technology and sonar's wavenumber ((wavenumber agorithm) algorithm, imaging through frequency domain algorithm, theoretically the imaging speed can be increased by $\mathrm{N}$ ( $\mathrm{N}$ is the number of elements) times. In addition, the effective element in the array is reduced[24]. The number, using sparse array technology to optimize the position and weighting coefficient of each effective element in the phased array transducer can also achieve the purpose of improving imaging efficiency. Compared with the improved post-processing imaging algorithm, this method has fewer interference factors, The principle is easy to understand, the cost can be reduced. And some classic array optimization methods can be used for reference, such as the genetic algorithm (GA) in the biological field, ant colony algorithm (ACA) and solid-based The simulated annealing algorithm (Simulated Annealing Algorithm, SAA) based on the annealing principle, etc. Wooh S et al. discussed the influencing factors of the main lobe, side lobe, and grating lobe in the ultrasonic phased array array pattern, combined with numerical analysis and experimental analysis to determine the sparse array The design of uniform sparse array is relatively simple, but the non-uniform sparse array and the uniform sparse array have their own characteristics. When designing the array, a reasonable choice should be made according to the actual clearing conditions[25]. The simulated annealing algorithm is used when designing the sparse array, The upper and lower limits of sidelobe values can be determined by numerical analysis methods, and the array performance is predictable[26]. TrUCCO uses this method to design a two-dimensional sparse array with expected sidelobe values, and has achieved good detection results in underwater three-dimensional imaging, But it is impossible to design a sparse array with arbitrary aperture and sparse rate. Compared with the randomly generated binary sequence, the one-dimensional sparse array based on the almost difference set has a lower peak sidelobe value. Haupt et al. took the lead Bring the biological genetic algorithm into the array design, through continuous crossover and mutation to find the optimal solution within the preset number of iterations[27]. Pliveri proposed a combination of Almost Deffirent Sets (ADS) and genetic algorithm The method designs a sparse array. This method can obtain a sparse array with better performance in fewer iterations, but the designed array is not used for actual detection. Yang Kai et al. used fuzzy particle swarm ptimizationagorithm (Fuzzy Particle swarm ptimizationagorithm, The FPSOA method optimizes the sparse antenna array and obtains good results. Xiang Ling fully derives the directivity function of the array sound beam, and analyzes the influencing factors of the main lobe, side lobe and grating lobe in the array directivity diagram, And design a non-uniform sparse array of cross transmitting and receiving, which can better suppress the side lobes of the array[28 29].

In recent research, as classic design methods are continuously improved and perfected, some more effective methods are continuously proposed[30]. For example, for the excitation signal of each element in the synthetic aperture technology

Specific modulation is performed, and the positions of the effective array elements used for transmission and reception are arranged by simulated annealing algorithm and weighted, which can improve the signal-to-noise ratio and detection speed of the detection result.Since the genetic algorithm is a random algorithm, the consistency is poor and it provides a sub-optimal sparse matrix column[31]. In this regard, Yang et al. applied the minimum redundancy method to the sparse array design, and the conjugator could design a sparse array with better performance. Chen Gang uses the ADS method to optimize the layout of each element in the large radar sparse array, which can achieve a higher array effect than the traditional method. The echo data received by the sparse array of phase correlation coefficients is used for post-processing imaging, which not only greatly reduces the side lobe and grating lobe levels of the array pattern, but also improves the lateral resolution and SNR of defects. At the same time, the phase discretization principle is applied to the sparse array design. Compared with the traditional wave forming method, the image spatial resolution and contrast resolution are improved[32].

ISSN: 0010-8189

(C) CONVERTER 2021

www.converter-magazine.info 


\section{Principle of all-focus imaging}

Ultrasonic phased array full matrix data uses the pulse repetition period of the exciting array element as the time interval to excite individual array elements in turn. After each array element is excited, the received signals of all the array elements are received and stored in the pulse repetition period. Thus, all array elements transmit-receive pair signals are obtained. Taking a one-dimensional linear phased array as an example, the number of array elements is $\mathrm{N}$, and the echo signal that excites the $\mathrm{i}$-th array element to be received by the $\mathrm{j}$ array element is defined as eij $(t), i, j=1,2, \ldots, N$, sequentially excites $N$ array elements, and each array element receives echo signals at the same time. The data collected in this way is called full matrix data. All-focus imaging is to perform postprocessing and imaging on the full-matrix data. For the commonly used wedge-coupled phased array detection, a coordinate system is established, the origin $\mathrm{O}$ is located at the center of the detection array, the $\mathrm{x}$-axis is to the right along the array direction, and the $\mathrm{z}$-axis is perpendicular to the array direction and points to the area to be tested. The number of phased array elements is $\mathrm{N}$, the width of the elements is a, the distance between the elements is $\mathrm{d}$, the sound velocity of the ultrasonic wave in the wedge and the workpiece are $\mathrm{c} 1$ and $\mathrm{c} 2$, and the height of the wedge is $\mathrm{H}$. For the element $\mathrm{i}$, the sound beam is at A certain incident point $\mathrm{Si}$ on the workpiece, F(x, z) is an arbitrary imaging point in the workpiece[33 34].

According to the principle of Fermat, the sound beam always travels along the path with the shortest time required. The sound beam propagation path from the $\mathrm{i}$-th element to the refraction point $\mathrm{Si}$ and then to the imaging point $\mathrm{F}$ can be determined, and the sound of the process can be obtained. The propagation time Ti is:

$$
\begin{gathered}
T_{i}=\min _{\left|x_{i}\right| \leq N d / 2,}\left(\frac{\sqrt{\left(x_{i}-x_{i t}\right)^{2}+H^{2}}}{c_{1}}+\frac{\sqrt{\left(x_{i t}-x\right)^{2}+(z-H)^{2}}}{c_{2}}\right)(1) \\
x=H \tan \theta_{i}+(z-H) \tan \theta_{s} \\
x_{i}=\left[\frac{N+1}{2}-i\right] d
\end{gathered}
$$

According to Snell's law:

$$
\frac{\sin \theta_{i}}{\sin \theta_{s}}=\frac{c_{1}}{c_{2}}(2)
$$

From equations (1), (2) and known quantities, the propagation time $\mathrm{Ti}$ of the sound beam emitted by the i-th array element to the imaging point $\mathrm{F}$ can be calculated. Similarly, the ultrasonic propagation time $\mathrm{Tj}$ of the sound beam reflected by the imaging point $F$ and received by the array element $\mathrm{j}$ can be obtained. Therefore, the propagation time $t \mathrm{ij}(\mathrm{x}, \mathrm{z})$ of the sound beam from the element $\mathrm{i}$ through the imaging point $\mathrm{F}$ to the element $\mathrm{j}$ is:

$$
\begin{gathered}
t_{i j}(x, z)=\frac{\sqrt{\left(x_{i}-x_{i t}\right)^{2}+H^{2}}+\sqrt{\left(x_{j}-x_{j r}\right)^{2}+H^{2}}}{c_{1}}+ \\
\frac{\sqrt{\left(x-x_{i t}\right)^{2}+(z-H)^{2}}+\sqrt{\left(x-x_{j r}\right)^{2}+(z-H)^{2}}}{c_{2}}
\end{gathered}
$$

In the formula, $x i$ and $x j$ represent the abscissas of the transmitting and receiving array elements, and xit and xjr are the abscissas of the refraction point of the transmitting and receiving sound beams at the interface respectively. According to the principle of reciprocity, the transmission and reception process is reversible. Using the law of time-delay superposition, superimpose the ultrasonic echo signals of all transmitting-receiving elements in the transducer passing through the F point, and the amplitude characterizing the imaging point can be obtained.

$$
I(x, z)=\sum_{i=1}^{N} \sum_{j=1}^{N} e_{i j}\left(t_{i j}(x, z)\right)(4)
$$

\section{Sparse total focus method}

\subsection{Related concepts of sparse total focus method}

The sparse all-focus method is to sparse the transmitting or receiving array, and perform imaging through incomplete sparse matrix data. The sparse array can be designed based on the effective aperture, so that the sparse array and the full array have the same effective aperture. Arrays with the same effective aperture have the same

ISSN: 0010-8189

(C) CONVERTER 2021

Www.converter-magazine.info 
point spread function (PSF) in the far-field area, and the point reflector is the imaging target. The imaging performance of the algorithm can be evaluated by the point spread function of the array.

Suppose there is a point scatterer in the measured medium, and r0 represents its position vector. In order to describe the position of the array element in the transmitting aperture and the receiving aperture, the binary functions $\mathrm{u}, \mathrm{v}$ of the imaging point position vector $\mathrm{r}$ are defined. These functions are non-zero fixed values at the transmitting and receiving elements, and 0 at other positions. Define wT and wR as the weight functions of the transmitting and receiving arrays, and the value of the function is only related to the position of the array element. The transmit aperture and receive aperture of the array can be obtained by performing spatial Fourier transform on the product of the array binary function and the weight function:

$$
\begin{aligned}
& A_{T}=\int_{-\infty}^{+\infty} u\left(r_{T}\right) \omega_{T}\left(r_{T}\right) \exp \left(k_{k b r}\right) d r_{T} \\
& A_{R}=\int_{-\infty}^{+\infty} v\left(r_{R}\right) \omega_{R}\left(r_{R}\right) \exp \left(k_{k} r_{R}\right) d r_{R}
\end{aligned}
$$

In the formula, $\mathrm{AT}$ and $\mathrm{AR}$ are the transmitting aperture and receiving aperture of the array respectively, and $\mathrm{k}$ is the wave number.

The point spread function includes the sound field distribution of all transmitting-receiving array elements at each point. By approximating the Green's function in the far field and using the phase shift technique, the point spread function of the far field area of the array can be obtained as:

$$
\begin{gathered}
i\left(r_{0}, r\right)=\frac{A^{2} \exp \left(2 i k\left(\left|r_{0}\right|-|r|\right)\right)}{\left|r_{0}\right|} A_{\mathrm{T}} k\left(\frac{r}{|r|}-\frac{r_{0}}{\left|r_{0}\right|}\right) \\
A_{\mathrm{R}} k\left(\frac{r}{|r|}-\frac{r_{0}}{\left|r_{0}\right|}\right)
\end{gathered}
$$

In the formula, $\mathrm{A}$ is a constant; $\mathrm{r} 0$ and $\mathrm{r}$ respectively represent the position vector of the point scatterer and the imaging point.

When correcting the sparse array, it is necessary to make the effective aperture consistent with the effective aperture of the full matrix. Therefore, there are many ways to define the weight function. For the sake of convenience, this article only considers the sparse transmitting array element. The receiving array element adopts the full array, and the weight wm of the transmitting array element is set to 1 . Only by adjusting the weight function $\mathrm{Rm}$ of the receiving array element, the sparse array and the full array have Same effective aperture. When the sparse transmitting array element is determined, the weight function $\mathrm{R}$ of the receiving array can be obtained according to equations (5) and (6). Finally, the sparse total focusing algorithm modified by the weight function can be obtained as:

$$
\begin{array}{r}
\square \square, \square=\square=\square \square \square \square=\square \square \square \boldsymbol{\omega} \square \square \square, \square \square \square \square \square, \square \square \square \square \mathbf{t} \square \square \square, \square \square \square \square I(x, z)= \\
\sum_{i=1}^{N_{\mathrm{T}}} \sum_{j=1}^{N_{\mathrm{R}}} \frac{\boldsymbol{\omega}_{\mathrm{T}}^{i}(\boldsymbol{x}, \mathbf{z}) \boldsymbol{\omega}_{\mathrm{R}}^{j}(\boldsymbol{x}, \mathbf{z}) \boldsymbol{e}_{i j}\left(\boldsymbol{t}_{i j}(\boldsymbol{x}, \mathbf{z})\right)}{\boldsymbol{C}_{i j}}(7)
\end{array}
$$

\subsection{Sparse all-focus imaging based on virtual source technology}

As shown in Figure 1, the one-dimensional linear phased array transducer is placed on the surface of the measured medium to establish a rectangular coordinate system. The $\mathrm{x}$-axis is parallel to the surface of the medium along the actual array direction, and the axis is perpendicular to the actual array and points to the specimen. Inside, the origin is at the center of the actual array. The focus delay law is used to excite the continuous $m$ array elements in the transducer. The excitation law is different, and the focus point position is also the same. As shown in Figure 2, the central array element is excited first, and then the two side array elements are excited in turn. It can be equivalent to a spherical wave emitted by a virtual source above the array, but the emitted energy is higher than the actual single array element; similarly, with the corresponding excitation The law excites the array element and also forms a virtual source below the actual array. The virtual source can emit spherical waves forward and backward as shown in Figure 2. Multiple virtual sources form a virtual source array, and the position of each virtual source in the virtual source array can be determined by the method introduced in section 3 .

ISSN: 0010-8189 

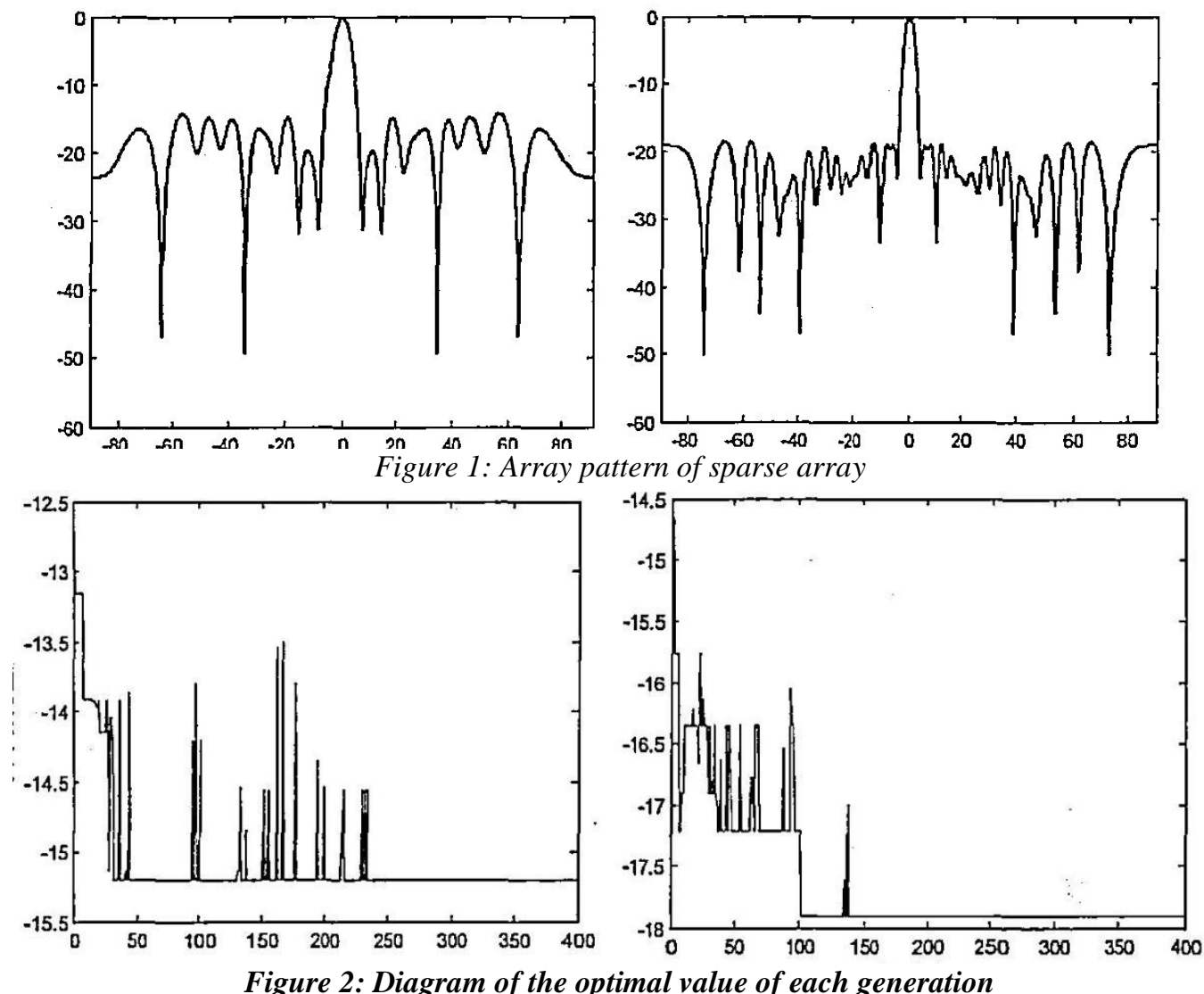

As mentioned earlier, the data used for imaging of a single virtual source is similar to the full-matrix data. Therefore, sparse all-focus imaging based on the virtual source needs to collect complete full-matrix data first, and select the corresponding array group data according to the location of the virtual source. Perform imaging. Ultrasonic phased array full-matrix data acquisition means that the ultrasonic phased array transducer with the number of array elements is $\mathrm{N}$, each array element is excited in turn, the first element is excited first, and all elements in the transducer receive echo signals. Then the second array element is excited, and all the array elements still receive the signal, until the Nth array element is excited, and all the array elements receive the signal. Place the one-dimensional linear phased array transducer on the surface of the measured medium to establish a rectangular coordinate system. The $\mathrm{x}$-axis is parallel to the surface of the medium along the actual array direction, the axis is perpendicular to the actual array and points to the inside of the test piece, and the origin is at the center of the actual array. The focus delay law is used to excite the continuous $m$ array elements in the transducer. The excitation law is different, and the focus point position is also the same. Excite the central array element first, and then the two side array elements in turn, which can be equivalent to a spherical wave emitted by a virtual source above the array, but the emission energy is higher than the actual single array element; similarly, the corresponding excitation rules are used to excite the array elements. A virtual source will be formed below the actual array, and the virtual source can emit spherical waves forward and backward. Multiple virtual sources form a virtual source array, and the position of each virtual source in the virtual source array can be determined.

\subsection{Optimize simulation results}

Taking a transducer with a total number of elements of 64 and a sparse array with an effective number of elements of 16 as an example, the genetic algorithm optimization of the sparse array layout is carried out. According to the reasonable parameter selection range, after many attempts, set the initial population set number to 40 , the iteration number to 204 , and the crossover probability and mutation probability to 0.8 and 0.05 respectively. Since the 
genetic algorithm is a random algorithm, in order to better compare After optimization, compared with the result before optimization, three sparse arrays of 8 elements are generated through the three-time genetic algorithm optimization, which are represented by A1, A2, and A3, respectively. Fig. 3 is a sparse array pattern of evenly distributed 8-array elements, and Fig. 4 is a pattern of three sparse arrays optimized by genetic algorithm. The performance indexes of their main lobe and side lobe are listed. It can be seen from the figure that, compared with the sparse array with 8 elements evenly distributed, the sparse array optimized by the genetic algorithm has a narrower main lobe width and a smaller peak value. Sidelobes, we can see that it has better imaging array characteristics. In addition, due to the characteristics of the main lobe and side lobe of the array, the effect of A1 is better than that of A2 and A3. Therefore, the sparse array designed by the genetic algorithm is used for imaging in the sparse array corresponding to A1.

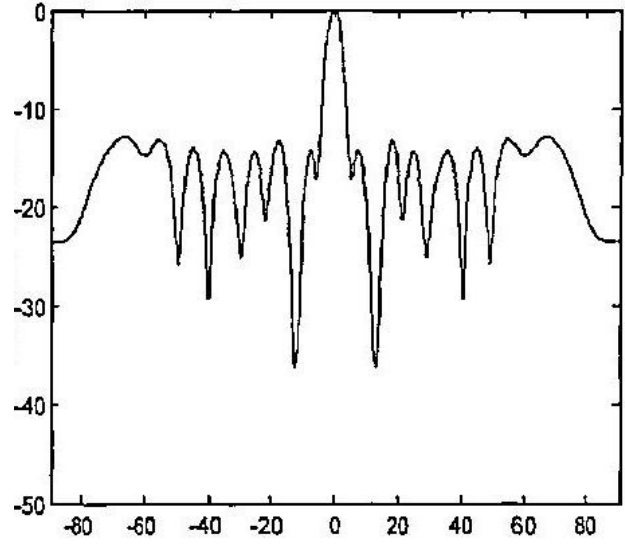

Figure 3.1: Uniform distribution

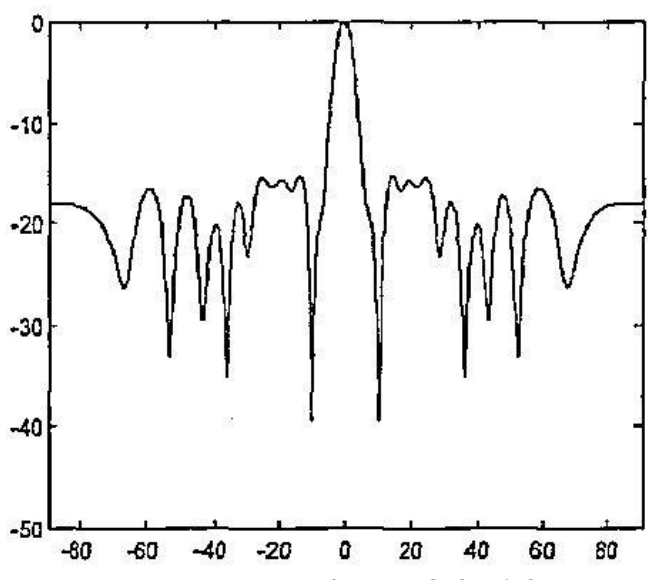

Figure 3.3: A2

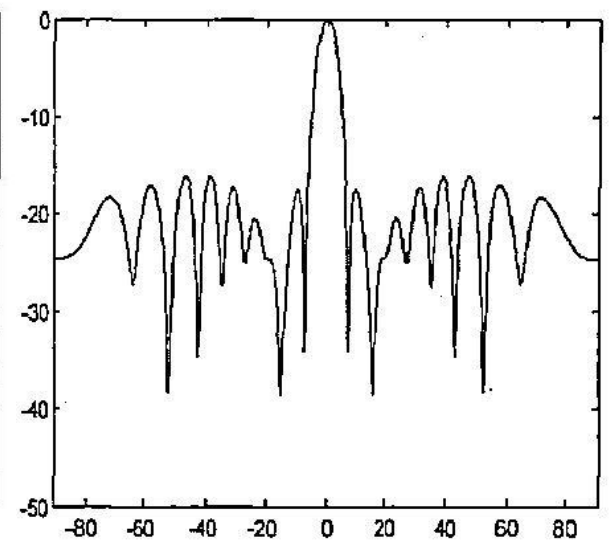

Figure 3.2: A1

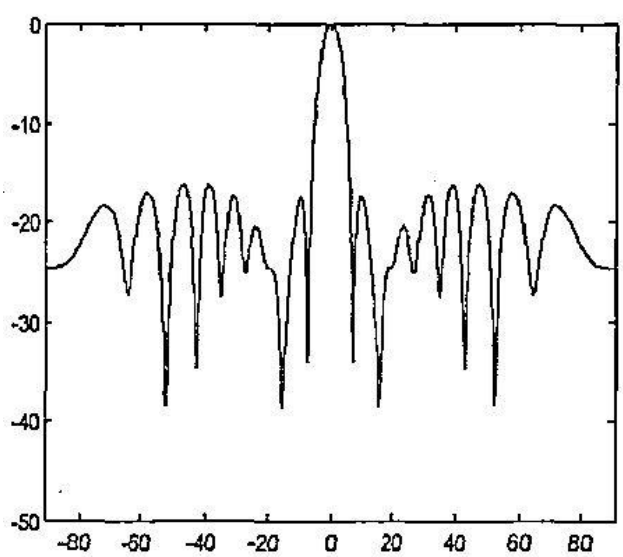

Figure 3.4: A3

Figure 3: Array directivity diagram of the rare earth array

The sparse all-focus method uses a designed sparse array to perform imaging by obtaining incomplete sparse matrix data. In order to make the imaging effect of the sparse array optimized based on the genetic algorithm close to the full array, the effective elements of the sparse array can be weighted to make the effective aperture of the sparse array consistent with the full array.

\section{Experimental results and discussion}

\subsection{Experimental results}

Take the circular arc-shaped side holes on the standard phased array B-type test block as the test object. As shown

ISSN: 0010-8189 
in Figure 4, the test area is marked by a white rectangular frame in the figure. There are 18 sides in this area with a diameter of $2 \mathrm{~mm}$. The holes are numbered 1-18 from top to bottom. The experiment uses the 5L32-0.6x 10 ultrasonic phased array transducer produced by Guangzhou Dople Electronic Technology Co., Ltd. and the SB 72NOL type 00 wedge. The total number of array elements in the transducer is 32 , and the distance between each array element The width of the array element is $0.5 \mathrm{~mm}$, the center frequency of the transducer is $5 \mathrm{MHz}$, the thickness of the wedge is $20 \mathrm{~mm}$, and the sound velocity of the ultrasonic wave in the wedge and the test block is $2730 \mathrm{~m} / \mathrm{s}$ and $5900 \mathrm{~m} / \mathrm{s}$, respectively. With a sampling frequency of $100 \mathrm{MHz}$, the full matrix data is collected and stored in accordance with the full matrix data collection method. The processor of the PC used for imaging is Core $\mathrm{i}$, and the running memory is $4 \mathrm{G}$. The imaging area is $75 \mathrm{~mm} * 60 \mathrm{~mm}$, and the horizontal and vertical imaging point spacing is $0.2 \mathrm{~mm}$. The imaging area is divided into a $376 \times 301$ pixel grid.
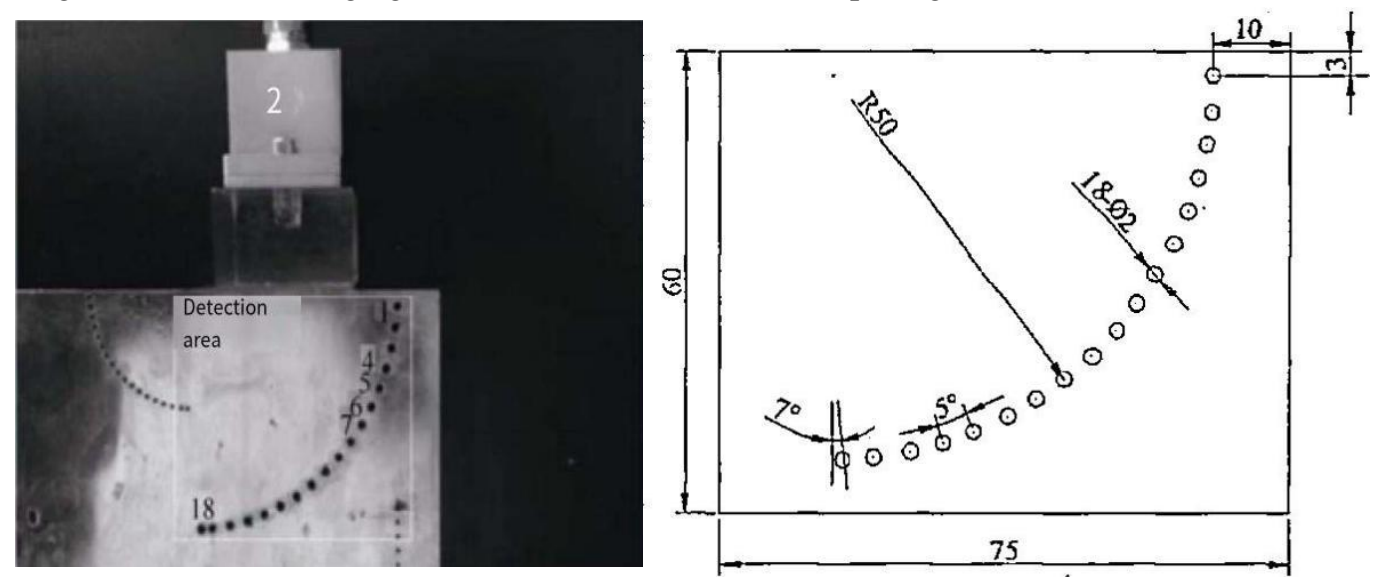

\section{(a) Test diagram (b) Dimensional drawing of the measured area of type phased array test block Figure 4: Ultrasonic phased array detection experiment}

Perform imaging according to the established medium full-focus algorithm, and express the imaging amplitude in decibels. The imaging result is shown in Figure 5. Figure 5a is a two-layer media full-focus imaging image of the entire detection area. The top and bottom of the image are the primary and secondary echoes of the wedge. Figure $5 \mathrm{~b}$ is an all-focus imaging image with the wedge echo removed. Since the two lowest defect holes coincide with the wedge secondary echo, the two lowest defect holes are not shown in Figure 5b. It can be seen that in order to avoid the influence of the wedge echo, it is necessary to select an appropriate wedge thickness according to the detection depth and the sound velocity of the medium during actual inspection. According to the number of sparse emission array elements set, the first element is the starting element of the sparse array, and the element with the largest lateral size of the sparse array is selected to form the detection aperture, and the effective elements are equally spaced and evenly distributed in the aperture. For example, when the number of sparse array elements is 8 , the number of effective elements is $1,5,9,13,17,21,25,29$.

Set the number of transmitting elements of the sparse matrix to 2, and the number of receiving elements to the number of full array elements of 32, and perform sparse all-focus imaging with 2 elements on the two layers of media, as shown in Figure 6. Figure $6 \mathrm{a}$ and Figure $6 \mathrm{~b}$ respectively show the uncorrected and corrected imaging images. It can be seen that the sparse all-focus imaging after the weight function correction has reduced side lobes and artifacts near the defect hole, and the imaging quality has been relatively improved. However, due to the small number of array elements, the overall imaging signal-to-noise ratio and resolution are insufficient.

ISSN: 0010-8189 


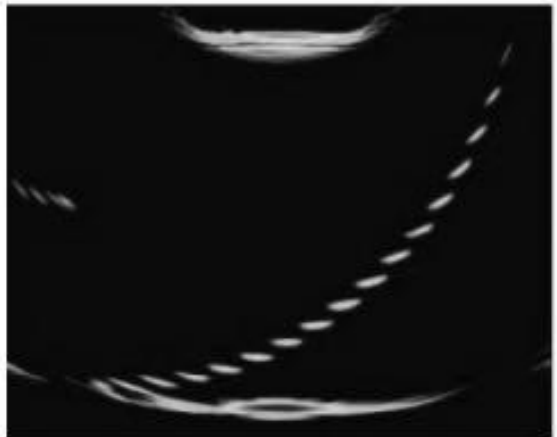

(a) Full focus imaging
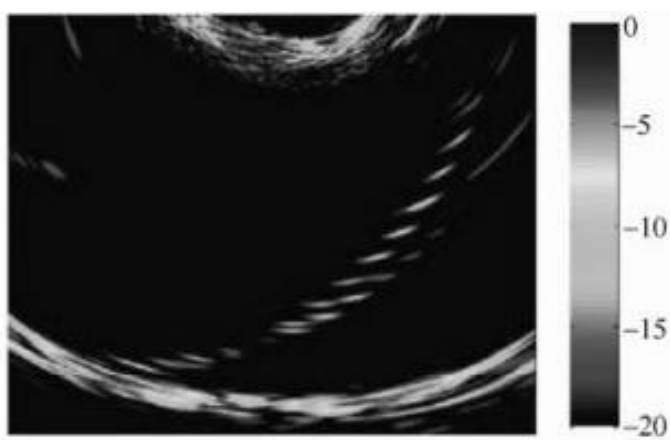
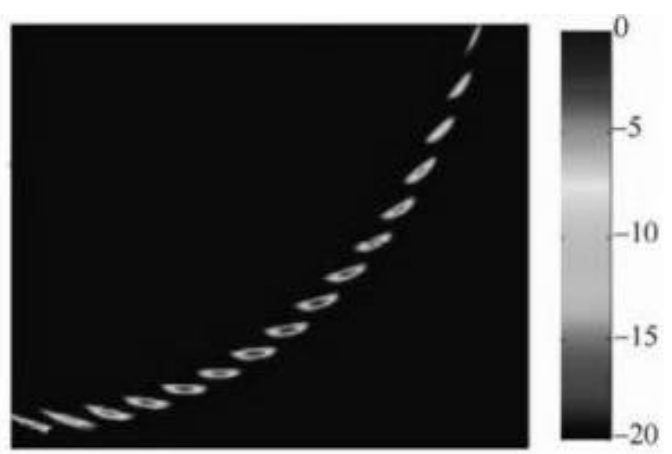

(b) Uniformly distributed all-focus imaging

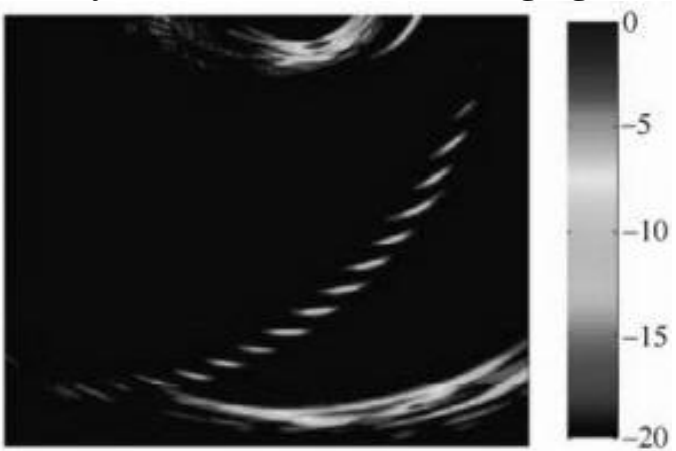

(c) Genetic algorithm optimization （d） Genetic algorithm optimization and modification Figure 5: Sparse all-focus imaging

\subsection{Discussion of results}

It can be seen from Figure 5 that the error value e of the sparse all-focus algorithm after the effective aperture correction is generally smaller than that of the uncorrected sparse all-focus algorithm, and the measurement result is closer to the full-matrix all-focus detection. As the number of sparse array elements increases, the value of $d$ gradually decreases, and the change is obvious at the beginning. With the continuous increase of the number of sparse array elements, the change tends to be gentle. When s elements are added, the error $d$ value between the modified full-focus algorithm and the full-matrix full-focus remains basically unchanged. In addition, using the full matrix data to perform a full-focus imaging in the corresponding defect hole area No. 4-7 takes 16.6 min. According to the data, when the number of sparse array elements reaches 8 , the $\mathrm{d}$ value of the modified sparse fullfocus method is all It can be controlled within $5.2 \%$. At this time, it only takes 4.2 minutes to perform a full-focus imaging, and the algorithm efficiency is increased by nearly 4 times.

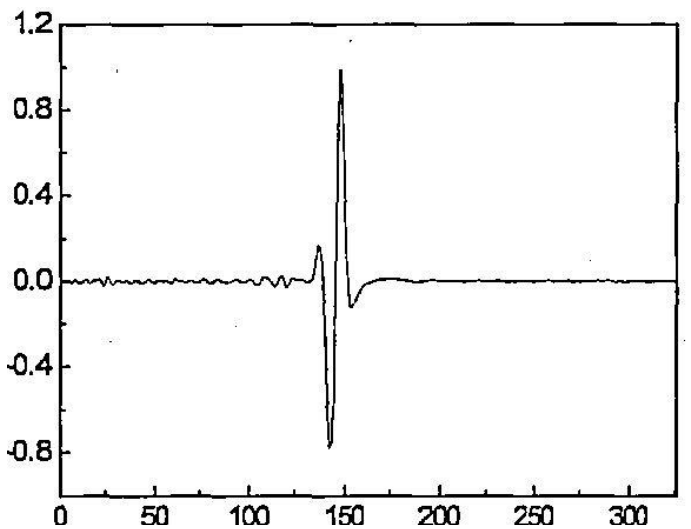

(a) Horizontal amplitude distribution

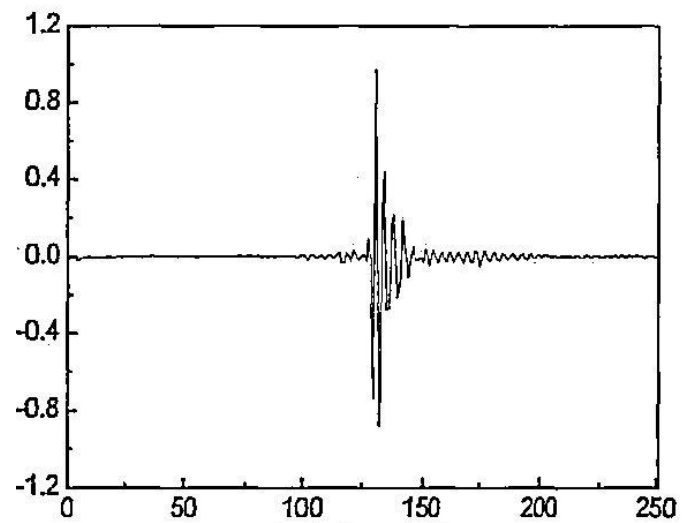

(b) Longitudinal amplitude distribution

ISSN: 0010-8189

(C) CONVERTER 2021

www.converter-magazine.info 

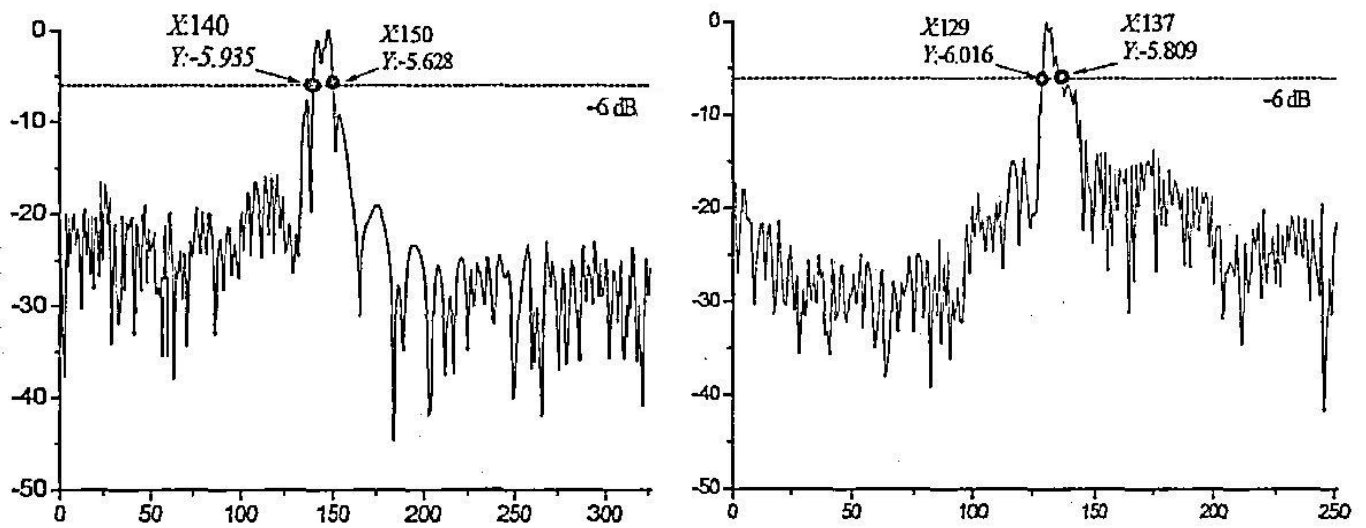

(c) Lateral sound pressure distribution

(d) Longitudinal sound pressure distribution

Figure 6: Horizontal and vertical sound pressure distribution of all-focus imaging

In order to extend the all-focus algorithm to the detection model of two-layer media and realize the all-focus imaging of the two-layer ultrasonic phased array, this chapter calculates the propagation path and propagation time of ultrasound in the two-layer medium based on the principle of acoustic Fermat. Two-layer media all-focus imaging model. On the other hand, in order to speed up the calculation efficiency of the all-focus algorithm, the sparse array optimized by the genetic algorithm is used, and the echo signals of the transmitter-receive array element are weighted through the concept of effective aperture, and a two-layer medium correction sparse all-focus imaging is established. model. Finally, through experimental analysis, it is verified that the established model can effectively improve the imaging efficiency of the all-focus method while ensuring the detection accuracy.

\section{Conclusion}

(1) Based on the Fermat principle, the delay time of each element in the two-layer medium is calculated, and the medium phased array all-focus imaging algorithm is established. In all-focus imaging, the area quantitative error is within 7\%, and the horizontal and vertical resolutions are both within one pixel interval. The effective imaging area is affected by the secondary echo of the wedge, and the depth of the wedge should be reasonably selected according to the detection depth and the sound velocity of the medium in actual inspection.

(2) Sparse the emission array of the medium full-focus algorithm, and establish a sparse full-focus algorithm based on effective aperture correction. This algorithm greatly improves the imaging efficiency of the algorithm under the premise of ensuring the imaging accuracy. For a 32-element transducer, when the sparse matrix data of 8 emission elements is used, the error value of the sparse matrix relative to the full matrix can be controlled within $5.2 \%$, and the algorithm efficiency is increased by nearly 4 times.

\section{References}

[1] Nonlinear ultrasonic phased array imaging.[J]. Potter J N,Croxford A J,Wilcox P D. Physical review letters. 2014 (14)

[2] Data set reduction for ultrasonic TFM imaging using the effective aperture approach and virtual sources[J]. S Bannouf,SRobert,OCasula,C Prada. Journal of Physics: Conference Series. 2013 (1)

[3] Effects of array transducer inconsistencies on total focusing method imaging performance[J]. JieZhang,Bruce W. Drinkwater,Paul D. Wilcox. NDT and E International. 2011 (4)

[4] Fully interleaved linear arrays with predictable sidelobes based on almost difference sets[J]. G. Oliveri,A. Massa. IET radar, sonar \& navigation. 2010 (5)

[5] Defect detection using ultrasonic arrays: The multi-mode total focusing method[J]. JieZhang,Bruce W. Drinkwater,Paul D. Wilcox,Alan J. Hunter. NDT and E International. 2009 (2)

[6] Linear Array Thinning Exploiting Almost Difference Sets[J]. Giacomo Oliveri,MassimoDonelli,Andrea Massa. IEEE Transactions on Antennas and Propagation. 2009 (12)

ISSN: 0010-8189 
[7] The wavenumber algorithm for full-matrix imaging using an ultrasonic array.[J]. Hunter Alan J,Drinkwater Bruce W,Wilcox Paul D. IEEE transactions on ultrasonics, ferroelectrics, and frequency control. 2008 (11)

[8] Sensitivity to point-spread function parameters in medical ultrasound image deconvolution[J] . HoChulShin,RichardPrager,JamesNg,HenryGomersall,NickKingsbury,GrahamTreece,Andrew Gee. Ultrasonics. 2008 (3)

[9] Advanced post-processing for scanned ultrasonic arrays: Application to defect detection and classification in non-destructive evaluation[J]. Caroline Holmes,Bruce W. Drinkwater,Paul D. Wilcox. Ultrasonics. 2008 (6)

[10] Finite Difference Time Domain Simulation of Ultrasonic Phased Array Sector Scan for Imaging Cracks in Large Pipes, Elbows, and Tee Sections[J]. L. Satyanarayan,K. V. Mohan,C. V. Krishnamurthy,K. Balasubramaniam. Research in Nondestructive Evaluation . 2008 (2)

[11] An implementation of synthetic aperture focusing technique in frequency domain.[J] . Stepinski Tadeusz. IEEE transactions on ultrasonics, ferroelectrics, and frequency control. 2007 (7)

[12] Ultrasonic arrays for non-destructive evaluation: A review[J] . Bruce W. Drinkwater,Paul D. Wilcox. NDT and E International. 2006 (7)

[13] A novel method to design sparse linear arrays for ultrasonic phased array[J] . Ping Yang,BinChen, KeRen Shi. Ultrasonics . 2006

[14] Post-processing of the full matrix of ultrasonic transmit-receive array data for non-destructive evaluation[J]. Caroline Holmes,Bruce W. Drinkwater,Paul D. Wilcox. NDT and E International. 2005 (8)

[15] Optimization of sparse synthetic transmit aperture imaging with coded excitation and frequency division[J]. Vera Behar,Dan Adam. Ultrasonics. 2005 (10)

[16] Particle Swarm Optimization Versus Genetic Algorithms for Phased Array Synthesis[J]. Daniel W. Boeringer,Douglas H. Werner. IEEE Transactions on Antennas and Propagation. 2004 (3)

[17] NDT techniques for railroad wheel and gauge corner inspection[J]. Rainer Pohl,AErhard,H.-J Montag,H.-M Thomas,HWüstenberg. NDT and E International. 2003 (2)

[18] Phased array element shapes for suppressing grating lobes.[J] . Pompei F Joseph,Wooh Shi-Chang. The Journal of the Acoustical Society of America. 2002 (5 Pt)

[19] Wavenumber domain reconstruction of SARSAS imagery using single transmitter and multiplereceiver geometry[J]. Callow H.J.,HayesM.P.,Gough P.T.. Electronics Letters. 2002 (7)

[20] Frequency-Wavenumber Migration of Ultrasonic Data[J]. Young-FoChang,Chir-CherngChern. Journal of Nondestructive Evaluation. 2000 (1)

[21] Full-focus imaging algorithm and calibration research in phased array ultrasonic testing technology[J]. Zhou Zhengqian,PengDi,LiYang,Hu Hongwei. Chinese Journal of Mechanical Engineering. 2015(10)

[22] Sparse optimization of multiple input and multiple output radar arrays using difference set theory[J]. Chen Gang, Gu Hong, SuWeimin, Bo Chao. Journal of Nanjing University of Science and Technology. 2013(04)

[23] A multi-constrained sparse wiring array antenna synthesis method [J]. Jia Weimin, Lin Zhiqiang, Yao Minli, Zhao Peng, Zhao Jianxun. Chinese Journal of Electronics. 2013(05)

[24] Simulation and optimization of sound field characteristics of sparse ultrasonic phased array[J]. Xiang Ling, Yang Tao. Computer simulation. 2013(03) 